\title{
The Influence of a New Uracil Derivative Carbicyl on Myocardium Bioenergetic Processes
}

\section{Iryna Nizhenkovska}

The department of pharmaceutical, biological and toxicological chemistry, Pharmaceutical Faculty, Bogomolets National Medical University, Kyiv, Ukraine

\section{Email address: \\ dekan-farm@ukr.net}

\section{To cite this article:}

Iryna Nizhenkovska. The Influence of a New Uracil Derivative Carbicyl on Myocardium Bioenergetic Processes. American Journal of Clinical and Experimental Medicine. Vol. 3, No. 4, 2015, pp. 162-166. doi: 10.11648/j.ajcem.20150304.16

\begin{abstract}
There was carried out research concerning the following metabolic deterioration: the metabolic disorders of nicotinamide nucleotides and such macro-energy compounds as adenyl nucleotides, creatine phosphate and their metabolism enzymes in myocardium tissue in case of anthracycline-induced toxic cardiomyopathy. The results of influence of a new physiologically active compound carbicyl that possesses metabolic effect on bioenergenic processes when used in rats with anthracycline-induced heart failure are presented comparing with other cardiotonic agents of different pharmaceutical classes. This study shows that the administration of a metabolic cardiotonic agent carbicyl to the rats that suffer from adriamycin-induced cardiomyopathy significantly normalizes the state of redox processes in myocardium. Carbicyl normalizes the parameters of mitochondrial function; it decreases the deterioration of energy metabolism in cardiomyocytes; carbicyl increases the content of the oxidized forms of NAD + and NADH+ coenzymes; and it increases the level of ATP, creatine phosphate and glycogen.
\end{abstract}

Keywords: Adriamycin, Carbicyl, Cardiotonic Agents, Cardiomyopathy

\section{Introduction}

Despite numerous studies of biochemical and molecular mechanisms of the deterioration of cardiomyocyte contractile function and its energy supply in conditions of heart failure, they are still poorly understood [1,2]; they are one of the most urgent problems of modern medicine. Existing literature data indicate the importance of searching and studying the mechanism of action of new cardiovascular drugs that possess cardioprotective properties among the analogs of such natural metabolites as: amino acids, nucleotides, physiologically active nitrogen-heterocyclic compounds [3].

Macrocyclic polyethers are a rather perspective class of substances; these compounds possess a high biological activity due to their unique complexing properties and similar structure with ionophore antibiotics [4]. Therefore, it was rationally to explore the influence of new representatives of crown ether derivatives in comparison with well-known cardiotonic agents.

To achieve this goal it was necessary:

1. to conduct a study of metabolic disorders of metabolism of such nicotinamide nucleotides and macroergic compounds as adenyl nucleotides, creatine phosphate and their metabolism enzymes in myocardium tissue in case of anthracycline-induced toxic cardiomyopathy;

2. to study the influence of a new physiologically active compound carbicyl that is a uracil derivative and possesses metabolic effect on the bioenergetic processes in myocardium of animals with anthracycline-induced toxic cardiomyopathy comparing with other nonsteroidal cardiotonic agent (dobutamine).

\section{Materials and Methods}

The investigations were conducted on adult Wistar rats (80). All manipulations with animals were carried out according to the European Convention on the Protection of Vertebrate Animals used for Experimental and other Scientific Purposes (Strasbourg, 1986). These animals were divided into 4 groups: 1 st group - the control group; 2 nd animals with experimental heart failure; 3 rd group - animals with experimental heart failure and that have been injected with carbicyl; 4th group - animals with experimental heart failure and that have been injected with nonsteroidal 
cardiotonic agent dobutamine. Experimental heart failure was established with intramuscular (i.m.) administration of adriamycin once a week at a dose of $5 \mathrm{mg} / \mathrm{kg}$ during 5 weeks to laboratory animals. Adriamycin hydrochloride was used in this experiment. Carbicyl was administrated i.m. at a dose of $250 \mathrm{mg} / \mathrm{kg}$ daily during 5 weeks. Nonsteroidal cardiotonic agent dobutamine was administrated i.m. at a dose of 10 $\mathrm{mg} / \mathrm{kg}$ daily also during 5 weeks.

In myocardium tissues the content of nicotinamide coenzymes (oxidized forms: nicotinamide adenine dinucleotide (NAD+) and nicotinamide adenine dinucleotide phosphate (NADP+); and reduced forms: nicotinamide adenine dinucleotide (NADH) and nicotinamide adenine dinucleotide phosphate (NADPH)) was determined using fluorometry; the activity of NAD-hydrolytic enzymes was determined by the rate of NAD+ hydrolysis [5], the content of adenine nucleotides was assessed using the electrophoretic method followed by spectrophotometry (the wavelength was 260 and $290 \mathrm{~nm}$ ). The content of creatine phosphate (CP) was determined as the difference between total and free creatine via spectrophotometry (the wavelength was $525 \mathrm{~nm}$ ); the glycogen content was evaluated with the help of the anthrone reagent; the activity of creatine phosphokinase (CPK) was determined by the formation of free creatine during the incubation with $\mathrm{CP}$ [6].

The upset of bioenergetic processes in myocardial cells in case of experimental cardiomyopathy has been found in many studies [7, 8]. However, there is no literature data concerning the cellular bioenergetic processes in myocardium during the experimental anthracycline-induced toxic cardiomyopathy that is realized by the administration of the cardiotoxic antibiotic adriamycin. That is why it became the subject of this research.

\section{Results and Discussion}

During this study it was observed that the administration of adriamycin in rats leads to the significant disruption of biological oxidation reactions and the delivery of energy substrates in cardiomyocytes. The evidence of such a deterioration is presented by negative dynamic in changes of the concentration and catabolism of such nicotinamide coenzymes such as NAD + and NADP + that are primary acceptors of electrons and protons in the electron transport chain in the mitochondrial membrane.

It was determined the significant (by 24.5\%) concentration decrease of the oxidized pyridine coenzymes in myocardial tissue and the total amount of oxidized and reduced coenzyme's forms in 1.5 time. This is a result of the limiting aerobic energy production in cells [9]. The activity of the enzymes that are responsible for the hydrolytic degradation (NAD+ glycohydrolase) was increased by $37.2 \%$ that can be regarded as one of the key biochemical mechanisms of reducing the concentration of nicotinamide nucleotides and therefore the deterioration of the biological oxidation processes in mitochondrial membranes. Apart from the biological oxidation connected with the oxidative phosphorylation process, in case of hypoxia and anaerobiosis the important substrates of energy metabolism for the permanent production of sufficient amount of ATP for the cardiomyocyte's functional activity are glycogen and CP.

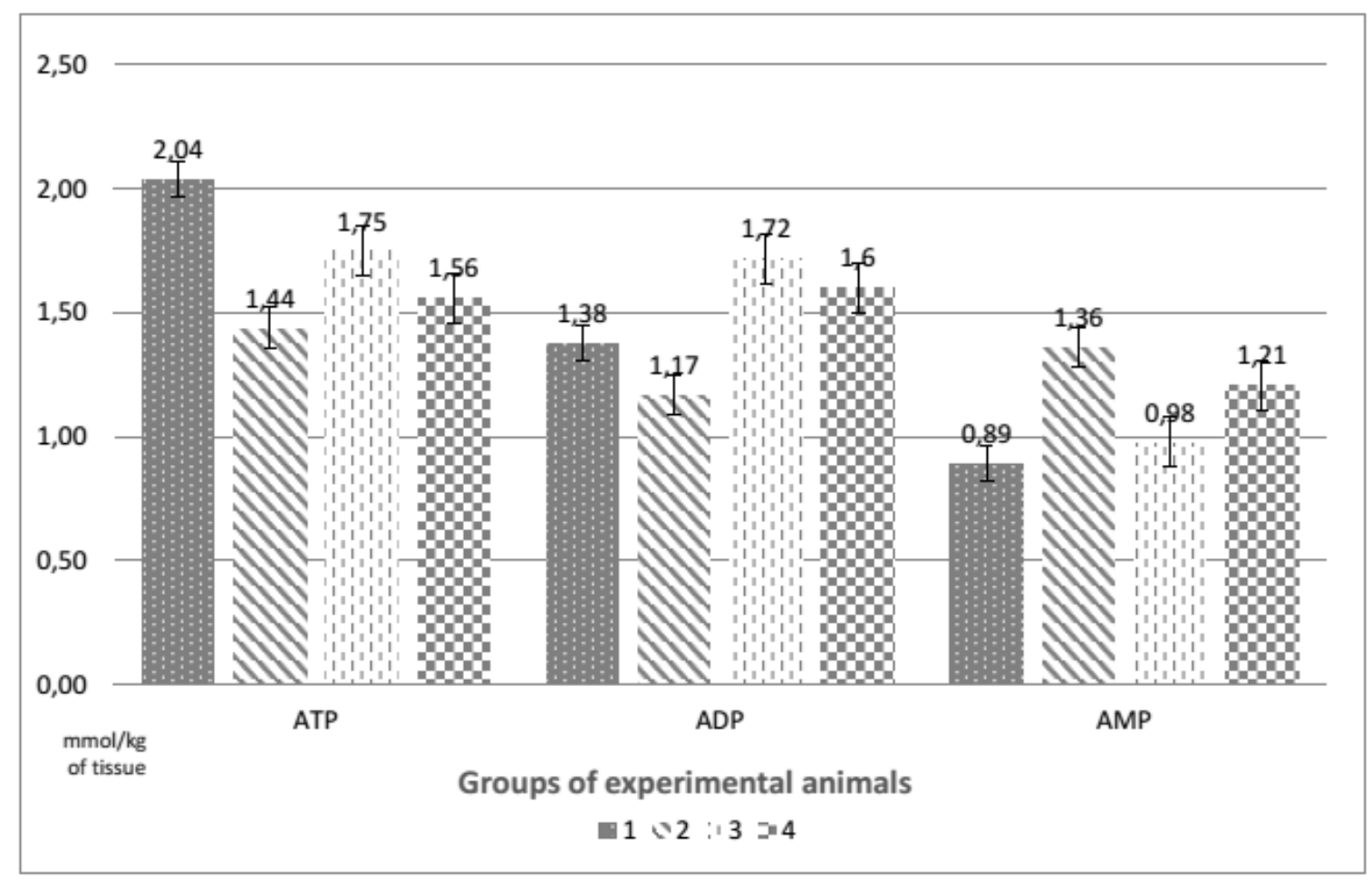

Figure 1. The carbicyl effect on parameters of adenyl nucleotides metabolism in myocardium of rats with adriamycin-induced cardiomyopathy 
Table 1. Changes of energy metabolism indicators in myocardium of rats with toxic anthracycline-induced cardiomyopathy ( $M \pm m)$.

\begin{tabular}{|c|c|c|}
\hline Energy metabolism indicators & Control $\left(1^{\text {st }}\right.$ group; $\left.n=10\right)$ & Adriamycin $\left(2^{\text {nd }}\right.$ group; $\left.n=10\right)$ \\
\hline $\mathrm{NAD}^{+}+\mathrm{NADP}^{+}, \mathrm{mcmol} / \mathrm{kg}$ of tissue & $483 \pm 7$ & $365 \pm 7 *$ \\
\hline $\mathrm{NAD}^{+}+\mathrm{NADP}^{+}+\mathrm{NADH}+\mathrm{NADPH}, \mathrm{mcmol} / \mathrm{kg}$ of tissue & $820 \pm 14$ & $764 \pm 7^{*}$ \\
\hline Ratio of oxidized/reduced forms of pyridine nucleotides & $1.43 \pm 0.04$ & $0.92 \pm 0.02 *$ \\
\hline NAD-hydrolase, mcmol/kg during 1 hour & $2615 \pm 84$ & $3689 \pm 86^{*}$ \\
\hline $\mathrm{CP}, \mathrm{mcmol} / \mathrm{g}$ of tissue & $3.94 \pm 0.88$ & $1.40 \pm 0.20^{*}$ \\
\hline $\mathrm{CPK}, \mathrm{mcmol} / \mathrm{g}$ of tissue during 1 minute & $5.75 \pm 0.22$ & $3.28 \pm 0.24^{*}$ \\
\hline Glycogen, $\mathrm{mg} / \mathrm{kg}$ of tissue & $218 \pm 13$ & $95 \pm 8^{*}$ \\
\hline
\end{tabular}

${ }^{*} \mathrm{p}<0.05$ concerning the control group

Table 2. Adenine nucleotide content (mmol/kg of tissue) in myocardium of rats with anthracycline-induced toxic cardiomyopathy $(M \pm m)$

\begin{tabular}{lll}
\hline Nucleotides & Control $\left(1^{\text {st }}\right.$ group $)$ & Adriamycin $\left(2^{\text {nd }}\right.$ group $)$ \\
\hline ATP & $2.04 \pm 0.07$ & $1.44 \pm 0.03^{*}$ \\
ADP & $1.38 \pm 0.08$ & $1.17 \pm 0.02^{*}$ \\
AMP & $0.89 \pm 0.06$ & $1.36 \pm 0.08^{*}$ \\
ATP/ADP & $1.47 \pm 0.05$ & $1.23 \pm 0.09^{*}$ \\
Phosphorylation Potential & $0.63 \pm 0.04$ & $0.51 \pm 0.04^{*}$ \\
\hline
\end{tabular}

${ }^{*} \mathrm{p}<0.05$ concerning the control group

Table 3. Influence of nonsteroidal cardiotonic agents on the CP and glycogen content and the CPK activity in myocardium of rats with adriamycin-induced cardiomyopathy $(M \pm m)$

\begin{tabular}{llll}
\hline Animal group & $\mathbf{C P}, \mathbf{~ m m o l} / \mathbf{g}$ & $\mathbf{C P K}, \mathbf{m c m o l} \mathbf{C P}$ per $\mathbf{1} \mathbf{g}$ tissue in $\mathbf{1 ~} \mathbf{m i n}$ & Glycogen, $\mathbf{m g} / \mathbf{k g}$ \\
\hline $1^{\text {st }}$ & $3.94 \pm 0.88$ & $5.75 \pm 0.22$ & $218.2 \pm 13.1$ \\
$2^{\text {nd }}$ & $1.4 \pm 0.2^{*}$ & $3.28 \pm 0.24^{*}$ & $95.2 \pm 8.0^{*}$ \\
$3^{\text {rd }}$ & $3.32 \pm 0.68^{* *}$ & $4.72 \pm 0.32^{* *}$ & $192.1 \pm 12.5^{* *}$ \\
$4^{\text {th }}$ & $1.9 \pm 0.54^{*}$ & $2.66 \pm 0.22$ & $98.3 \pm 11.2^{*}$ \\
\hline
\end{tabular}

${ }^{*} \mathrm{p}<0.05$ concerning the control group

$* * \mathrm{p}<0.05$ concerning the $2^{\text {nd }}$ group

Table 4. The carbicyl effect on the level of nicotinamide coenzymes and the NAD-hyrdolase activity in myocardium of rats with adriamycin-induced cardiomyopathy

\begin{tabular}{llll}
\hline Animal group & NAD+ + NADP+, mcmol/kg of tissue & NAD-hydrolase, mcmol/kg & $\begin{array}{l}\text { Ratio of oxidized/reduced forms of pyridine } \\
\text { nucleotides }\end{array}$ \\
\hline $1^{\text {st }}$ & $483 \pm 7$ & $2615 \pm 84$ & $1.43 \pm 0.04$ \\
$2^{\text {nd }}$ & $365 \pm 7^{*}$ & $3689 \pm 86^{*}$ & $0.92 \pm 0.02^{*}$ \\
$3^{\text {rd }}$ & $456 \pm 11^{* *}$ & $2898 \pm 56^{* *}$ & $1.26 \pm 0.06^{* *}$ \\
$4^{\text {th }}$ & $386 \pm 12^{*}$ & $3618 \pm 53^{*}$ & $0.96 \pm 0.04^{*}$ \\
\hline
\end{tabular}

${ }^{*} \mathrm{p}<0.05$ concerning the control group

$* * \mathrm{p}<0.05$ concerning the $2^{\text {nd }}$ group

The significance of CP that serves as a rapidly mobilizable reserve of high-energy phosphates for the ATP production due to the creatine kinase enzyme reaction is well established in many experimental studies $[6,10,11]$.

According to the obtained data (see Table 1) the 5-week administration of adriamycin leads to a significant reduction of the glycogen level (by 56\%) in comparison with the control group. The $\mathrm{CP}$ content was also decreased in the second group in 2.8 times and was accompanied by the CPK decrease by $43 \%$ concerning the control group. The changes in the amount of glycogen and creatine kinase system's components are common for the toxic damages of the heart muscle, including the anthracyclines administration. Anthracyclines specifically bind to DNA by intercalating between the base pairs of the double helix, and thus the inhibitory action of DNA-dependent RNA polymerase is disrupted; and the intracellular regeneration processes of cardiomyocytes are deteriorated [12].

The sharp decrease of CP content and the decreased activity of the CPK is one of the key mechanism of the imbalance of the normal physiological replenishment of ATP store that is necessary for the actin-myosin contraction function under the 
conditions of pathology

In order to evaluate the cardioprotective effects of a new physiologically active compound carbicyl in comparison with the beta-adrenergic agonist such as dobutamine it was carried out a study of the influence of the above mentioned physiologically active compounds on such metabolism indicators as nicotinamide nucleotides, the content of adenine nucleotides, glycogen and the state of creatine kinase system in case of adriamycin-induced toxic cardiomyopathy. According to the obtained data in those rats that were administered carbicyl during the adriamycin administration the content of oxidized forms of nicotinamide nucleotides was increased by $24.9 \%$. The oxidized/reduced coefficient of pyridine nucleotides during the carbicyl administration had been increasing by $36.9 \%$. The total amount of coenzymes was also increased and thus indicating the tendency of the normalization of oxidative processes. The NAD-hydrolase activity had been normalized during the experiment.

According to the data presented in Table 2 the adenylate pool reacts to the prolonged anthracycline antibiotic administration by the decreased level of ATP $(-29.5 \%)$, the ATP/ADP ratio decrease $(-16.5 \%)$ and the decreased phosphorylation potential (- 19.1\%).

By reducing the use of inorganic phosphate in the phosphorylation reactions, the amount of inorganic phosphate increases in myocardium of animals by $30.8 \%$.

The simulated anthracycline cardiomyopathy is accompanied by a significant decrease in functional performance of the left ventricle, and is characterized by significant violations of energy metabolism in the heart muscle, as evidenced by the data of the presented experiments.

These data are entirely consistent with the morphological studies of myocardial tissue in case of anthracycline heart failure [13] and suggests that the deterioration of the energy supply system can lead to the defeats of subcellular mechanisms responsible for the act of contraction-relaxation, and be the cause of further deterioration of the pathological process. Among the possible biochemical mechanisms responsible for the formation of cardiomyopathy under the influence of anthracycline antibiotics may be the effect of these pharmacological agents on the system of $\mathrm{Ca}^{2+}$ transport across membranes of cardiomyocytes. However, we should not forget that the relaxation of the myocardium is also an energy-intensive process as sarcoplasmic reticulum $\mathrm{Ca}^{2+}$ ATPase participates in this process, as well as significant amounts of ATP are necessary for the interaction of the last with the heads of the myosin filaments and the rupture of covalent bonds in actin-myosin complexes that are responsible for the relaxation process.

This is due to the high dependence of the process of relaxation of cardiomyocytes from phosphorylation potential [9], which also causes contribute to the violation of the functional properties of cardiac muscle in heart failure. In addition, there is evidence that adriamycin blocks proton-pumped NADH oxidase that participates in the synthesis of intracellular messengers, performing in the cell signaling function, namely involved in carrying out the core of a proliferative signal required for the activation of tyrosine kinases and activates the cascade of phosphorylation of membrane and cytosolic proteins of cardiomyocytes.

The influence of carbicyl and dobutamine on metabolism indicators of adenine nucleotides of rats in case of anthracycline-induced toxic cardiomyopathy is presented in Figure 1.

The obtained from this research data indicated that the adenine nucleotide system in myocardium of rats that were administered adriamycin also underwent fewer deteriorations: the ATP content was increased by $21.5 \%$; the phosphorylation potential was increased by $13.7 \%$; the AMP level was decreased by $28 \%$ concerning the group of animals that suffered from anthracycline-induced toxic cardiomyopathy; the content of inorganic phosphorus was approaching the values of the control group.

As it was indicated above, the important biochemical components of cardiomyocytes, the reserves of which have been significantly decreased under the condition of anthracycline-induced toxic cardiomyopathy had been glycogen and CPK; the latter one functions as a transporter under the action of CK is enzymes in the reactions of ATP synthesis. The results of study of the metabolic drug carbicyl influence in comparison with the beta-adrenergic agonist such as dobutamine on the content of glycogen and CK and on the CPK activity in myocardium of rats with anthracycline-induced toxic cardiomyopathy that was caused by adriamycin (see Table 3) indicate that the use of carbicyl increases the decreased concentrations of glycogen and CPK caused by adriamycin. Particularly the level of glycogen in myocardial tissue of animals that were administered carbicyl during the treatment with adriamycin exceeded the corresponding values in rats that were treated with adriamycin without metabolic drug; the glycogen content by $102 \%$ approached the control values.

Such significant protective effect of carbicyl was established in respect to $\mathrm{CP}$ concentrations, to CPK activity in the myocardium of rats with adriamycin-induced intoxication.

It should be emphasized that the tested compound carbicyl that possesses metabolic effects and has cardiotonic activity significantly activates the processes of energy production and the intensity of metabolism of myocardium of intact rats; moreover it significantly normalizes deteriorations in the system of macro-energy phosphates and nicotinamide coenzymes in case of anthracycline-induced toxic cardiomyopathy (see Table 4). Considerable improvements of the energy metabolism of myocardium in case of carbicyl use can probably be explained by its chemical structure that is similar to pyrimidine bases which due to the influence on the synthesis of nucleic acids and other regulatory molecules may affect the plastic processes in cells and processes associated with energy production.

\section{Conclusions}

1. During this study there were investigated biochemical mechanisms of the deterioration of bioenergetics 
processes in cardiomyocytes in terms of anthracycline-induced toxic cardiomyopathy; this deterioration was presented by the upset of oxidative processes in mytochondria, by the decrease of the reserves of metabolic energy and such macro-energy compounds as glycogen, creatine phosphate, ATP and ADP that are biochemical substrates of the energy supply realized by myofibrillar actomyosin complex in case of heart failure.

2. For the first time it was established that the administration to rats with anthracycline-induced toxic cardiomyopathy a new metabolic cardiotonic carbicyl that is chemically a crown ether derivative significantly normalizes the redox processes in myocardium; it reduces the severity of energy metabolism disorders in cardiomyocytes; it increases the content of the oxidized forms of coenzymes NAD+ and NADP + , increases ATP levels, CPK as well as glycogen.

\section{References}

[1] Korkushko, O. and Lishnevskaya, V. 2003, J. Blood Circulation and Hemostasis, $1,8$.

[2] Frolkis, V., Bezrukov, V. and Kulchitskiy, O. 1994, Aging and Experimental Aging Cardiovascular Pathology, Kyiv.
[3] Nizhenkovskaya, I. 2013, J. Current Topics in Pharmacology, 17(1), 103.

[4] Shinkai, S. 1985, J. Journal of the D.I. Mendeleev All-union Chemical Society, 30(5), 546.

[5] Telepneva, V. and Isayeva, I. 1967, J. Medical Chemistry Questions, 13, 242.

[6] Chmielewski, Yu., Gubskiy, Yu and Zaitseva, S. 1985, Biolodical Chemistry. Practical Work Book, Kyiv.

[7] Ivashkin, V., Gorbatenkova, O. and Drapkina, O. 2004, J. Clinical Medicine, 82(2), 20.

[8] Mokhort, N., Horchakova, N. and Hrynevych, A. 1999, Pharmacodynamics and the Search of New Cardiotonic Drugs, Kyiv.

[9] Boldyrev, A. 1982, J. Biological Sciences, 4, 5.

[10] Nizhenkovska, I., Gladchuk, A., Yanitska L. 2013, J. Medical Chemistry, 15 (2), 77.

[11] Saks, V., Javadov, S., Pozyn, E. 1989, Phosphocreatine: biochemical and pharmacological action and clinical use, Moscow.

[12] Vatutin N., Kalinkina, N., Katynh, E. 1999, J. Ukrainian Journal of Chemotherapy, 2 (32), 11.

[13] Semenova, L., Nepomnyashchii, L., Semenov, D. 1985, Morphology of heart muscle cells in case of plastic failure, The Scientific Thought, Novosibirsk. 